\section{The Increasing Role of Immunotherapy in the Management of Solid Cancers and its impact on Caribbean health services resources and provision.}

Dr. Jo-Anne Kissoon

\section{Correspondence to: \\ Dr. Jo-anne Kissoon \\ Gerontology Unit, St. James Medical Complex \\ Email: jo-annekissoon@hotmail.com}

\section{BACKGROUND}

In the Caribbean, cancer is the second leading cause of death, affecting one in every five persons. ${ }^{1,2}$ Of even greater concern is the mortality rate for breast and prostate cancer in Caribbean countries which is amongst the highest in the world. In Antigua and Barbuda for example, the mortality rate for these cancers is 44.7 per hundred thousand as opposed to the United States where it is $15.4 .^{2}$ These figures are indicative of the challenges faced by health services in providing effective cancer treatment for the developing countries of the Caribbean. Noted deficiencies include the need for more qualified oncologists operating within the Caribbean region as evidenced by a large number of patients with cancer seeking access to the public health care systems. ${ }^{3}$

Another major challenge is the unavailability of cancer medication, the cost of which is prohibitive, often placing it outside the reach of the average citizen. Thus, there is a reliance on what many perceive to be an under-funded and failing public health care system to provide the necessary treatment and support. Those with the financial resources seek treatment abroad or in one of the larger islands. ${ }^{2}$ Therefore, in the current environment, a diagnosis of cancer presents a major challenge for patients. In the Caribbean, the more common cancers include breast, cervical, colon, prostate and lung cancer. ${ }^{2}$. These cancers are usually treated using a combination of conventional therapies such as surgery, radiation and chemotherapy ${ }^{2}$

Recently, immunotherapy has been considered one of the more promising medical advances in cancer treatment. Immunotherapy drugs have a different side effect profile from traditional treatments and have promising outcomes for skin, breast, lung, kidney, head and neck cancers and Hodgkin's lymphoma. ${ }^{4}$ In the Caribbean, one advantage of these drugs is increased survival rates in melanoma patients with brain metastases, patients with triple-negative breast cancer, prostate cancer and patients with metastatic lung cancer. ${ }^{5,6}$ The adaptation of immunotherapy drugs may also be beneficial in the treatment of SARS-Cov- $2^{7}$ Thus, health services in the Caribbean should consider the acquisition of immunotherapy drugs as a major benefit in improving survival rates.

The decision to invest in immunotherapy drugs should consider the disadvantages of the treatment. The drugs may not work for all patients; others may develop immune related adverse events (irAEs) such as colitis, rash, pneumonitis, myalgia and hypothyroidism. ${ }^{8}$ Patient responses to immunotherapy drugs have also proven unpredictable and consequently response rates remain unpredictable. One forecast for the future of Immunotherapy is as follows ${ }^{8}$ :

"An improvement in the efficacy of immunotherapies will likely involve a more personalized and multi modal approach that cannot only target specific antigens that are present in the patient's tumor but is supplemented with agents such as epigenetic inhibitors and microbiota enhancers to elicit a more robust response."

Currently, health services struggle to meet the increasing need for the provision of cancer treatment. Factored into this struggle would be the high cost of available treatment options 
with immunotherapy ranking amongst the highest and the competing needs of those with other medical conditions. The case for immunotherapy is strong; however, the decision whether it is a worthwhile investment for the Caribbean will ultimately depend upon budgets and priorities.

\section{THE CONVENTIONAL APPROACH TO THE MANAGEMENT OF CANCER}

The National Cancer Institute defines solid cancer as 'an abnormal mass of tissue that usually does not contain cysts or liquid areas'. ${ }^{9}$ There are several types of solid cancers, such as sarcomas, carcinomas and lymphomas which are classified according to their cell composition. Traditional approaches to the management of solid cancer have included a combination of surgery, chemotherapy and radiation, all of which have assisted in combating the disease.

The factors that determine the course of treatment are the type and stage of solid cancer as well as the cost of treatment. However, it is ultimately the patient's decision that would determine the course of treatment. The traditional modalities such as surgery, chemotherapy and radiation therapy all have restrictions on their application but there have been improvements which support better cancer management and a more acceptable quality of life during treatment.

\section{Surgical Options}

Surgery is a treatment option that may be considered at every stage of cancer; however, there are conflicting views as to the role of surgery in metastatic cancer. Some oncologists still include surgery as a possibility for the removal of metastases in certain circumstances. The majority, however, see the trauma and inflammation associated with surgery as possibly enhancing tumor growth, increasing the establishment of new metastases and accelerating growth of residual and micro metastatic disease. ${ }^{10}$ Surgery may also be excluded as a treatment option in managing solid cancers when the tumours are large and invasive. It may also be inappropriate when the patient is elderly or has poor performance status, as survival is less likely. Other limitations would include risk of damage to nearby tissue, the possibility of not removing all tumour cells and additional complications such as infection. ${ }^{11}$ The most recent advance in cancer surgery has been robot-assisted surgery. This recent advancement decreases the risk of infection and damage to surrounding tissue. The resulting surgery is more precise; however, it is expensive and must be performed by a doctor with the required skillset. $^{12}$

\section{Chemotherapy}

Chemotherapy targets actively replicating cancer cells and its effects are systemic. Therefore the side effect profile of this group of drugs is extensive and includes nausea and vomiting, hair loss, possible permanent infertility and secondary malignancy. Other limitations of this group are "drug-resistance by tumors, poor penetration by the drug and inadequate drug dosing".13

This treatment modality, which is normally combined with others, has also seen advances over the past decade, with modern chemotherapy drugs now concentrating on reducing toxicity and associated side effects. Current drug combinations are also more targeted and more effective. $^{14}$

\section{Radiotherapy}

Radiotherapy mainly involves ionizing radiation which inevitably causes injury or damage to the surrounding tissue. It can be used as a primary tool, neoadjuvant, adjuvant, combination or even in the palliative setting. Toxic effects of radiation also include painful mouth ulcers, cataracts, alopecia, increased pigmentation and secondary malignancies.

Recent advances in technology have also improved the way this treatment modality is delivered. There is now intensity-modulated radio- 
therapy which is seen as an improvement in 3D conformal radiation therapy. It is a move away from conventional usage where dosage distribution is the result of trial and error. A more exacting approach, this computer-driven technology allows for the delivery of uniquely tailored dose variations within the radiation

field. ${ }^{15}$ Stereotactic body radiotherapy and particle beam therapy are also gaining momentum.

Given these limitations, there is a continuous search to discover other treatment options for solid cancers. One of the more promising developments has been immunotherapy, which has been revolutionizing solid cancer treatment as early as $1891 .{ }^{16}$ The appeal of immunotherapy, therefore, is that it uses a different mechanism which ultimately overcomes some of the limitations faced by traditional treatment modalities.

\section{THE MECHANICS OF IMMUNOTHERAPY}

Immunotherapy as a new form of cancer treatment is unique as it stimulates and strengthens the body's innate immune system to fight cancer. It does this by utilising products, either present in the body or laboratory-made, to improve or restore immune system function. It can also put a brake on signals from cancer cells that work to suppress the immune system. ${ }^{17}$

The recent strides that have been made in the management of solid cancers through immunotherapy have been due to a better understanding of its mechanism of action. ${ }^{18}$ This new concept of "tumour immune editing" was put forward by Schreiber and col-

leagues. ${ }^{19}$ Immunotherapy itself branches off into several different types including therapeutic vaccines, monoclonal antibodies, checkpoint inhibitors and cytokines.

\section{Therapeutic vaccines}

These immunotherapy vaccines differ from the conventional HPV and Hepatitis B cancer vaccines which are aimed at the prevention of cervical cancer and some liver cancers. They work through the "enhancement or stimulation" of the body's immune system to fight against solid cancers. These vaccines are however reported to have low clinical efficacy and sometimes used in tandem with other immunotherapies or targeted therapies. ${ }^{20}$

\section{Monoclonal antibodies}

These very specific antibodies can provoke an immune response which results in cell death. Thus, they are particularly successful in treating colorectal as well as head and neck cancers. Their success rate is especially good when introduced into a programme with other conventional modalities such as chemotherapy or radiotherapy. This group is also able to prevent tumour cell growth by "triggering an immunemediated cytotoxic response". ${ }^{17}$

\section{Checkpoint inhibitors}

One of the more common types of immunotherapy is checkpoint inhibitors. These drugs work by inhibiting the cancer cells that attempt to block or stop the immune cells of the human body from going into action. This interaction between the T-cells and cancer cells is through receptors or checkpoints called CTLA-4, PD-L1 or PD-1. Ultimately, they allow cancer-fighting T-cells of the immune system to recognize the cancer cells, which then enables them to attack those cancer cells. ${ }^{21}$

\section{Cytokines}

Cytokines are proteins produced by certain cells within the immune system. Cytokines such as Interleukin 2 (IL-2) and Interferon - a (IFN - a) stimulate the immune system through the mass production of T-cells. They work well in cases of renal cell carcinoma and melanoma.

\section{ADVANTAGES AND DISADVANTAGES OF IM- MUNOTHERAPY}

In the fight against cancer, this relatively new group of drugs called immunotherapy has brought a ray of hope, especially in the lives of patients coping with advanced (metastatic) 
lung and skin cancer.

In the case of melanoma, the figures show that checkpoint blockade immunotherapy more than doubled survival rates from 5.2 months to 12.4 months. Additionally, the four-year survival rate for persons receiving immunotherapy stood at around 28.1 per cent, a marked difference from the $11.1 \%$ for those who did not. No other treatment modality has been this successful, especially in the treatment of skin cancers. $^{22}$

Immunotherapy also works in tandem with other treatment modalities such as chemotherapy to improve the management of advanced nonsmall cell lung carcinoma. ${ }^{23}$ Two of the major positive aspects of immunotherapy are its good side effect profile and its immunomemory which is a unique advantage relative to other treatment modalities. Thus, if cancer recurs after being treated with immunotherapy, the immune system recognizes it and resumes its attack which results in an extended cancerfree period. This treatment is also more specific in terms of its target, thereby sparing the healthy cells from destruction. ${ }^{23}$

Immunotherapy works by harnessing and enhancing the innate immune system to work against cancer cells. Therefore, the process is not fast and has its peculiarity which is called "pseudoprogression". ${ }^{18}$ This unique phenomenon is explained by the T-cells interacting with cancer cells causing temporary swelling in approximately $10 \%$ of patients who receive this treatment which then subsides as cancer cells die.

As with all other treatment modalities, immunotherapy has its limitations. When checkpoint inhibitors are introduced there is the possibility of immune-related adverse events (irAEs). ${ }^{23,24}$ Common side effects include fatigue which affects about a quarter of its users, diarrhoea secondary to colitis, dyspnea and cough secondary to pneumonitis, dermatitis, hepatitis and hypothyroidism. Some of the side effects such as heart palpitations, weight gain, and diarrhoea tend to subside after the first treatment. ${ }^{23}$

There are also times when solid tumours respond initially to immunotherapy and then stop, resulting in tumour recurrence. Unfortunately, immunotherapy is not effective for all patients. Less than $50 \%$ of the patients who are treated with immunotherapy respond to it. ${ }^{25}$ Thus, there may be uncertainty in selecting the appropriate target group as the target population with the most likelihood of success would only be persons carrying certain biomarkers, testing for which is also costly.

At present, research has focused on the use of tumor-infiltrating lymphocytes and PD-L1 expression within the tumor microenvironment as predictors of immune response in patients undergoing immunotherapy. Similar consideration is also being given to a larger panel of gene signatures, chemokines and other factors that correlate with patient response. ${ }^{26}$

\section{ABILITY OF HEALTH SERVICES TO PROVIDE IMMUNOTHERAPY SERVICES}

Governments throughout the Caribbean face differing challenges in providing health services and in particular cancer treatments. As summed up in a recent series on cancer control in the Caribbean ${ }^{2}$

"These island nations all face similar challenges of a growing cancer burden, inadequate cancer surveillance, geographically isolated small populations, vulnerable ecological and economic situations, insufficient resources, poor access to screening and treatment, and overburdened health-care systems."

The 2014 PAHO/WHO document on Health Financing in the Americas proposed a benchmark of $6 \%$ for public expenditure on health as a percentage of gross domestic product or GDP. ${ }^{27}$ Recent figures show that the countries of the Caribbean have lagged in that regard. Data for the year 2016 shows Cuba as having 
allocated the highest percentage of GDP on public health. ${ }^{28}$ This country spent more than twice the $\mathrm{PAHO} / \mathrm{WHO}$ suggested allocation at $12.19 \%$. Other smaller economies allocated much less, with Guyana having a total allocation of $4.24 \%$, followed by Saint Vincent and the Grenadines with a total allocation of $3.56 \%$.

According to the World Bank data, Caribbean countries with a higher than 6\% GDP contribution to public healthcare for that year, were Barbados with a $6.96 \%$ allocation, followed by Trinidad and Tobago at $6.51 \%$ and the Bahamas at $6.38 \%$. The other countries reaching the benchmark were Belize at a score of $6.12 \%$, Jamaica with $6.07 \%$ public health/GDP expenditure and Suriname at $6.06 \%$ comparative spending. ${ }^{28}$

Further, according to the 2019 data only three countries, Jamaica, Martinique and Trinidad and Tobago, had National Cancer Plans in place, with Suriname having a draft only. ${ }^{2}$ Similarly, concerning national screening programs, only Martinique offered screening for cervical cancer, breast cancer and bowel cancer. St Lucia organized screening programmes for cervical cancer, and breast cancer, while Jamaica had a national screening programme for cervical cancer only. No such established national screening programmes were present in any of the other islands. ${ }^{2}$

Another challenge for the island economies has been the presence of oncology services to meet the needs of its patients. A 2018 study on the state of Caribbean oncology services which excluded the countries of The Bahamas, Guyana, St. Lucia and Haiti summarized the position of the region as follows. ${ }^{3}$ :

"Oncology services were insufficient in the majority of countries. Haematology proved to be the most adequately staffed with six countries (60\%) having the recommended number of specialists. Medical oncology services were deficient in five countries (50\%). Radiation oncology services were the most limited with nine countries (90\%) unable to provide the required quota of specialists. The majority of the workforce consisted of nonnationals (55\%). The remaining practitioners were nationals, and of these $50 \%$ were regionally trained. Oncological care was primarily offered within the public sector."

Other challenges which the region faces include the unavailability of medication within the public system as current foreign exchange and funding restrictions make it almost impossible to import certain cancer drugs. The task is made even more difficult by the absence of trained personnel, surgeons and specialists such as oncologists. ${ }^{3}$

Developed countries also face challenges and with the advent of immunotherapy and its associated costs the situation is no less complicated. Recent outbreaks such as the Coronavirus pandemic and older issues such as HIV prevention and support, which stand to affect large parts of the population, often take priority.

In the Caribbean there appears to be no public health service institutions that offer immunotherapy treatment except for Cuba, which has made significant progress in developing its brand of immune therapies. ${ }^{29}$

\section{FUTURE PLANS}

As countries worldwide are faced with ageing populations, increased prevalence of cancer and limited resources, the economic burden faced by the health care services is even more challenging. Immunotherapy has increased survival rates and made the treatment of solid cancers more effective and tolerable, thereby improving the quality of life for patients, mainly in the developed world. The benefits of immunotherapy in the fight against cancer are significant but do not extend to developing nations. According to WHO data, approximately $70 \%$ of deaths from cancer occur in developing nations. ${ }^{30}$ These cancer deaths would be due to several factors including excess tobacco and alcohol use, increasing obesity along with a 
similarly increased sedentary lifestyle, carcinogenic infections and financial constraints.

In the developing countries of the Caribbean, the cost of traditional cancer treatments such as surgery, chemotherapy and radiotherapy already pose an economic challenge for the public healthcare system. The cost of immunotherapy alone and more commonly, in combination with other modalities is quoted in articles as approximately US\$100,000 annually per patient. ${ }^{31,32}$ This places the treatment out of the present and future reach of most health care systems. ${ }^{31,32}$ Therefore, the high cost of immunotherapy would place an even greater burden on an already underfunded Caribbean public healthcare system.

Immunotherapy has increased survival rates and made the treatment of solid cancer more effective and tolerable in patients with advanced non-small cell lung cancer (NSCLC). This treatment has extended patient survival by five years. Similarly, the survival rate for younger patients with melanoma has also witnessed an increase due to the introduction of immunotherapy. ${ }^{33,34}$

For the Caribbean to reduce the economic burden, the focus in the fight against cancer should be on the development of prevention campaigns, including educating the population on the avoidance of risk factors. Specific importance should also be placed on vaccination against HPV and Hepatitis B viruses, as this has the potential to prevent one million cancer cases annually as well as the introduction of anti-smoking campaigns in countries where there are none. ${ }^{30}$

The central issue for health service providers in both developed and developing nations must be that of ensuring equality of access to these lifesaving cancer treatments amongst their populations. This becomes a balancing act between affordable pricing and guaranteed returns to ensure continued research, which may not be easily achieved. Perhaps we can learn from the Cuban experience of a small economy providing free immunotherapy treatment to its citizens, while simultaneously investing in research and the development of immunotherapy drugs. ${ }^{23}$ This dream may only be achieved when all stakeholders such as governments, pharmaceutical research companies and health service providers work together to improve the quality of care for the people they serve.

Ethical Approval statement: Not applicable

Informed consent statement: Not applicable

Conflict of interest: none declared

Funding statement: None

\section{References}

1. Warner WA, Lee TY, Badal $\mathrm{K}$ et al. Cancer Incidence and Mortality Rates and Trends in Trinidad and Tobago. BMC Cancer $2018 \mathrm{https} / / /$ bmccancer.biomedcentral.com/ articles/10.1186/s12885-018-4625$x$ (Accessed 29/01/2020)

2. Spence $D$, Dyer $R$, Andall-Brereton $G$ et al. Cancer control in Small Island Nations 3, The Lancet Oncology, 2019 pages 1-19

3. Alleyne Mike K. The Caribbean Community Clinical Oncology Workforce: Analyzing Where We Are Today and Projecting for Tomorrow; Journal of Oncology, Volume 2018 https://www.hindawi.com/journals/ jo/2018/7286281/ (Accessed 29/01/2020)

4. Lacombe S. Cancer Immunotherapy, British Society for Immunology - Policy Briefing, December 2016 https:// www.immunology.org/sites/default/files/ Cancer\%20Immunotherapy.pdf (Accessed $16 / 03 / 2020$ )

5. Brigham and Women's Hospital. Immunotherapy doubles survival rates for patients 
with melanoma brain metastases: Checkpoint inhibitors increase median survival to 12.4 months, and more than double percentage of patients who survive 4 years or longer. ScienceDaily

2018. www.sciencedaily.com/

releas-

es/2018/07/180712100541.htm (Accessed $29 / 01 / 2020$ )

6. Heady D. Immunotherapy improves fiveyear survival rate of people with advanced lung cancer. Medicalxpress, June 3, 2019 https://medicalxpress.com/ news/2019-06-immunotherapy-five-yearsurvival-people-advanced.html (Accessed $16 / 03 / 20$ )

7. De Wint G. CEL-SCI Initiates Development of Immunotherapy to Treat COVID-19 Coronavirus Infection. Businesswire, March 09, 2020 https://www.businesswire.com/news/ home/20200309005285/en/ (Accessed $17 / 03 / 2020$ )

8. Sambi M, Bagheri L, Szewczuk R. Current Challenges in Cancer Immunotherapy: Multimodal Approaches to Improve Efficacy and Patient Response Rates, Journal of Oncology, Volume 2019https://www.hindawi.com/ journals/jo/2019/4508794/ (Accessed $17 / 03 / 2020$ )

9. National Institutes of Health. National Cancer Institute Dictionary of Cancer Terms.

Solid Tumor, 2020. https:// www.cancer.gov/publications/dictionaries/ cancer-terms/def/solid-tumor (Accessed 29/01/2020)

10. Tohme S, Simmons RL, Tsung A. Surgery for Cancer: A Trigger for Metastases. Cancer Res. 2017 Apr 1; 77(7): 1548-1552. https:// www.ncbi.nlm.nih.gov/pmc/articles/ PMC5380551/ (Accessed 29/01/2020)

11. Winship Cancer Institute. Cancerquest. Cancer Treatments, 2019https:// www.cancerquest.org/patients/ treatments (Accessed 29/01/2020)

12. Martin LJ. Cancer Treatment Breakthroughs. Web MD. November 2018https:// www.webmd.com/cancer/detect-treatcancer-17/advances-cancertreatment (Accessed 29/01/2020)

13. Gavhane Y N, Shete A, Bhagat AK et al. Solid Tumors: Facts, Challenges and Solutions. International Journal of Pharma Sciences and Research (IJPSR) Vol. 2 (1), 2011, pages 1-12. http://www.ijpsr.info/docs/ IJPSR11-02-01-01.pdf (Accessed 29/01/2020)

14. Celgene Corporation. Chemotherapy: What's Old Is New Again - Advancements in Chemotherapy for Cancer; January 2014 https:// www.celgene.com/chemotherapytreatment/ (Accessed 29/01/2020)

15. Garibaldi C, Jereczek-Fossa BA, Marvaso G et al. Recent advances in radiation oncology. ecancermedicalscience $2017 \mathrm{Vol}$ 11 https://www.ncbi.nlm.nih.gov/pmc/ articles/PMC5718253/ (Accessed 29/01/2020)

16. Mc Carthy EF. The Toxins of William B. Coley and the Treatment of Bone and Soft Tissue Sarcomas. lowa Orthopaedic Journal. 2006 Vol. 26: pages 154 - 158. https:// www.ncbi.nlm.nih.gov/pmc/articles/ PMC1888599/ (Accessed 29/01/2020)

17. American Society of Clinical Oncology. Understanding Immunotherapy, 2019 https:// www.cancer.net/sites/cancer.net/files/ asco_answers_immunotherapy.pdf (Accessed 29/01/2020)

18. Filipovic A. The Role of Immunotherapy in treating Solid Cancers. Grandround, Cancer World. Jan/Feb 2017.https:// cancerworld.net/e-grandround/the-role-ofimmunotherapy-in-treating-solidcancers/ (Accessed 29/01/2020) 
19. Menon S, Shin S, Dy G. Advances in Cancer Immunotherapy in Solid Tumors. Cancers 2016, Vol. 8 (12) page 106 https:// www.ncbi.nlm.nih.gov/pmc/articles/ PMC5187504/ (Accessed 29/01/2020)

20. Mougel A, Terme M, Tanchot C. Therapeutic Cancer Vaccine and combinations with antiangiogenic therapies and immune checkpoint blockade. Frontiers in Immunology. 2019 10:467. https://www.frontiersin.org/ articles/10.3389/fimmu.2019.00467/ full (Accessed 29/01/2020)

21. Dranitsaris G, Zhu X, Adunlin G,Vincent MD. Cost effectiveness vs. affordability in the age of immuno-oncology cancer drugs. Expert Review of Pharmacoeconomics and Outcomes Research. April 2018; Vol 18 Issue 4 . https://www.tandfonline.com/doi/ abs/10.1080/14737167.2018.1467270 (Acce ssed $29 / 01 / 2020$ )

22. Zhou $Y$, Chen $C$, Zhang $X$ et al. Immune checkpoint Inhibitor plus chemotherapy versus conventional chemotherapy for first line treatment in advanced non-small cell lung carcinoma: a systematic review and metaanalysis. Journal for Immunotherapy Cancer 2018; Vol 6: 155 https:// www.ncbi.nlm.nih.gov/ pubmed/30577837 (Accessed 29/01/2020)

23.Pathak N. Pros and Cons of immunotherapy. Web MD. January 2019 https:// www.webmd.com/cancer/immunotherapyrisks-benefits (Accessed 29/01/2020)

24. Trinh S, Le A, Gowani S et al. Management of Immune-Related Adverse Events Associated with Immune Checkpoint Inhibitor Therapy: a Minireview of Current Clinical Guidelines. Asia-Pacific Journal of Oncology Nursing, 2019 Apr-Jun; 6(2): 154160 https://www.ncbi.nlm.nih.gov/pmc/ articles/PMC6371672/ (Accessed $18 / 03 / 2020$ )
25. Conolly C, Bambhania K, Naidoo J. Immune -Related Adverse Events: A Case-Based Approach. Frontiers in Oncology 2018; 9:530. https://www.frontiersin.org/ articles/10.3389/fonc. 2019.00530/ full (Accessed 29/01/2020)

26. Spencer K, Wang J, Silk A et al. Biomarkers for Immunotherapy: Current Developments and Challenges. American Society of Clinical Oncology - Educational Book, 2016 https://www.ncbi.nlm.nih.gov/ pubmed/27249758

27. 27. PAHO/WHO. Health financing in the Americas, 2017 https://www.paho.org/ salud-en-las-americas-2017/? $p=178$ (Accessed 29/01/2020)

28. 28. The World Bank. Current health expenditure (\% of GDP) - Latin America \& Caribbean, 2016 https:// data.worldbank.org/indicator/ SH.XPD.CHEX.GD.ZS? locations=ZJ (Accessed 29/01/2020)

29. Sansom C. Cuban Cancer Immunotherapy. ecancer News. March 2010 https:// ecancer.org/news/3077-cuban-cancerimmunotherapy.php (Accessed 29/01/2020)

30. WHO. Cancer Fact Sheet.September 2018 https://www.who.int/news-room/fact - sheets/detail/cancer (Accessed 29/01/2020)

31. Belluz J. The Nobel Prize is a reminder of the outrageous cost of curing cancer: Cancer's most promising therapies come with $\$ 100,000$-plus price tags. VOX 2018 https://www.vox.com/science-andhealth/2018/10/1/17923720/ immunotherapy-cancer-cost (Accessed 29/01/2020)

32. Pietrangelo A. The Value and Cost of Immunotherapy Cancer Treatments. Healthline 2016 https:// 
www.healthline.com/health-news/value-and -cost-of-immunotherapy (Accessed

$29 / 01 / 2020$ )

33. Goodman A. Studies Report Prolonged LongTerm Survival with Immunotherapy vs Chemotherapy in Advanced NSCLC, The ASCO

Post. October 2019. https:// www.ascopost.com/issues/october-102019/prolonged-long-term-survival-withimmunotherapy-vs-chemo-in-advancednsclc/ (Accessed 17/03/19)

34. Sternberg A. Boost in Survival Rates for Younger Patients with Melanoma Attributed to Immunotherapy Agents. Targeted Oncology. September 2019https:// www.targetedonc.com/publications/targeted -therapy-news/2019/september-2019/boost -in-survival-rates-for-younger-patients-with -melanoma-attributed-to-immunotherapyagents 